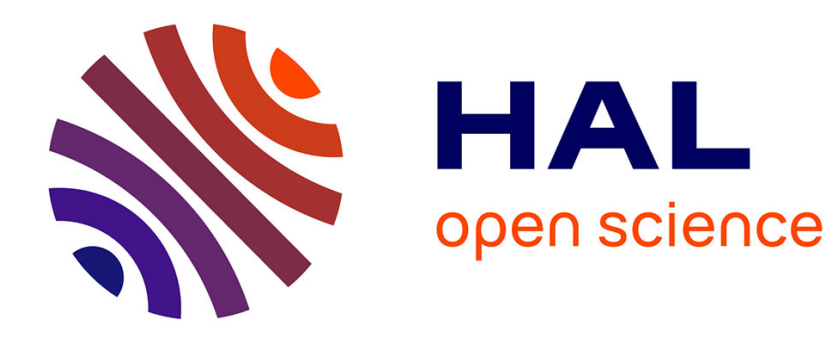

\title{
Generalized Collective Excitations in Supercritical Argon
}

Taras Bryk, Giancarlo Ruocco

\section{To cite this version:}

Taras Bryk, Giancarlo Ruocco. Generalized Collective Excitations in Supercritical Argon. Molecular Physics, 2011, pp.1. 10.1080/00268976.2011.617321 . hal-00745614

\section{HAL Id: hal-00745614 https://hal.science/hal-00745614}

Submitted on 26 Oct 2012

HAL is a multi-disciplinary open access archive for the deposit and dissemination of scientific research documents, whether they are published or not. The documents may come from teaching and research institutions in France or abroad, or from public or private research centers.
L'archive ouverte pluridisciplinaire HAL, est destinée au dépôt et à la diffusion de documents scientifiques de niveau recherche, publiés ou non, émanant des établissements d'enseignement et de recherche français ou étrangers, des laboratoires publics ou privés. 


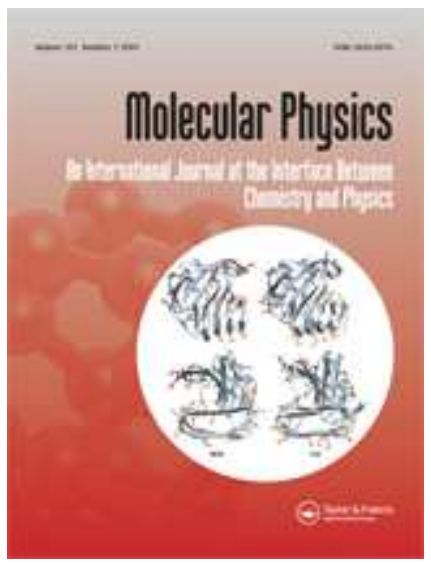

\section{Generalized Collective Excitations in Supercritical Argon}

\begin{tabular}{|r|l|}
\hline Journal: & Molecular Physics \\
\hline Manuscript ID: & TMPH-2011-0242.R1 \\
\hline Manuscript Type: & Special Issue in honour of Luciano Reatto \\
\hline Date Submitted by the & 19-Aug-2011 \\
\hline $\begin{array}{l}\text { Complete List of Authors: } \\
\text { Keywords: }\end{array}$ & $\begin{array}{l}\text { Bryk, Taras; Institute for Condensed Matter Physics, National } \\
\text { Academy of Sciences of Ukraine } \\
\text { Ruocco, Giancarlo; Universita di } \\
\text { Roma La Sapienza, Dipartimento di Fisica }\end{array}$ \\
\hline $\begin{array}{l}\text { dynics, structural relaxation } \\
\text { Note: The following files were submitted by the author for peer review, but cannot be converted } \\
\text { to PDF. You must view these files (e.g. movies) online. }\end{array}$ \\
\hline $\begin{array}{l}\text { a3.tex } \\
\text { figures.zip }\end{array}$ \\
\hline
\end{tabular}

\section{SCHOLARONE" \\ Manuscripts}


Molecular Physics

Vol. 00, No. 00, Month 2009, 1-10

\title{
RESEARCH ARTICLE
}

\section{Generalized Collective Excitations in Supercritical Argon}

\author{
Taras Bryk ${ }^{a, b}$ and Giancarlo Ruocco ${ }^{c, d *}$ \\ ${ }^{a}$ Institute for Condensed Matter Physics, National Academy of Sciences of Ukraine, 1 \\ Svientsitskii Street, 79011 Lviv, Ukraine; ${ }^{b}$ Institute of Applied Mathematics and \\ Fundamental Sciences, National Technical University of Lviv, 79013 Lviv, Ukraine; \\ ${ }^{c}$ Dipartimento di Fisica, Universita di Roma La Sapienza, I-00185, Roma, Italy; \\ ${ }^{d}$ IPCF-CNR, Universita di Roma, I-00185, Roma, Italy \\ (Received 00 Month 200x; final version received 00 Month 200x)
}

\begin{abstract}
Dispersion and damping of collective excitations in supercritical Argon along isothermal line of $280 \mathrm{~K}$ are studied by a combination of molecular dynamics simulations and Generalized Collective Mode (GCM) analysis. "Positive dispersion" of collective excitations and its dependence on density is discussed on the basis of GCM theory of "positive dispersion".
\end{abstract}

\begin{abstract}
Keywords: supercritical fluids; collective excitations; dispersion law; molecular dynamics; generalized collective modes; structural relaxation
\end{abstract}

\section{Introduction}

Collective dynamics in liquids and supercritical fluids is reviving an essential increase of interest of scientific community. Systematic improvements of resolution of Inelastic X-ray Scattering (IXS) and Inelastic Neutron Scattering (INS) techniques $[1,2]$ made possible precise estimations of dispersion of collective excitations in liquids as well as search for manifestation of non-hydrodynamic collective processes in dynamic structure factors $S(k, \omega)$, where $k$ and $\omega$ are wave number and frequency, respectively. Very recently one of the most fascination problems in collective dynamics of supercritical fluids has become the issue of dependence of so-called "positive dispersion" of collective excitations on density. Right outside the hydrodynamic regime the sound excitations in dense fluids have dispersion $\omega_{s}(k)$ not linear with wave number anymore but bent up towards higher frequencies. This "positive dispersion" of collective excitations was initially explained by mode coupling theory $(\mathrm{MCT})[3,4]$. The microscopic mechanism responsible for "positive dispersion" in MCT [3] is the non-local (with different wave numbers) coupling of hydrodynamic relaxation and propagating modes. The MCT yields a correction to the hydrodynamic dispersion law of acoustic excitations $\alpha_{s} k^{5 / 2}$ with a positive prefactor $\alpha_{s}$. This prefactor can be calculated from a sophisticated expression given in Ref.[5], that requires the knowledge of explicit density dependences of adiabatic speed of sound $c_{s}(n)$ and thermal expansion coefficient $\alpha_{T}(n)$.

Recent IXS experiments performed on supercritical Ar at $573 \mathrm{~K}$ and subsequent MD simulations [6] showed that the positive dispersion in supercritical Ar was

*Corresponding author. Email: Giancarlo.Ruocco@roma1.infn.it 
decreasing with reduction of density and almost vanished at some density, that was associated with extended into supercritical region Widom line [7]. One of the consequences of this finding was a suggestion that the "positive dispersion" can be a dynamic quantity that can discriminate between liquid-like and gas-like fluids in the supercritical region. However the calculations of density dependence of the MCT coefficient $\alpha_{s}$ for Ar at $573 \mathrm{~K}$ that is responsible for the "positive dispersion" were not in agreement with experimental findings, but supported previous calculations [5] for Ar at $120 \mathrm{~K}$ indicating increase of $\alpha_{s}$ with decreasing density.

Recently a theory of "positive dispersion" of collective excitations in fluids was developed within the method of Generalized Collective Modes (GCM) [8]. The essential difference between MCT and GCM is in explicit treatment of nonhydrodynamic processes in GCM approach and their coupling with hydrodynamic modes in local approximation $[9,10]$. Namely due to coupling of collective excitations with a non-hydrodynamic process of structural relaxation one obtains within the GCM analytical treatment the "positive dispersion" of acoustic excitations on the boundary of hydrodynamic regime [8]. Another interesting finding was the possibility of "negative dispersion" of acoustic excitations due to their coupling with thermal processes. The GCM analysis of collective modes was performed in Ref.[8] for liquid Ar simulated with Lennard-Jones potentials at two temperatures $205 \mathrm{~K}$ and $573 \mathrm{~K}$. It was shown that the "positive dispersion" vanishes with decrease of density for both temperatures, while "negative dispersion" can appear for the temperatures and densities by approaching the critical point.

An important issue is to study the "positive" dispersion of collective excitations along more isothermal lines in the supercritical region. This would either support or discard the suggestion that the "positive dispersion" vanishes by approaching the Widom line from the high-density side. Besides, a problem in simulations of dynamic properties of supercritical fluids close to critical point are finite-size effects. In [8] for the lower isothermal line corresponding to the temperature of $205 \mathrm{~K}$ was obtained a large deviation from the NIST data for the ratio of specific heats $\gamma$ right at its maximum as a function of density, that can be a consequence of finite-size effects. Therefore in this study we are aimed to check the relevant thermodynamic quantities obtained from the simulations with the NIST data in order to be sure that our simulations correctly reflect thermal processes in the supercritical fluid and then perform theoretical analysis of "positive dispersion" based on GCM approach. The remaining paper is organized as follows: in next section we give the details of molecular dynamics simulations and consequent GCM analysis. The results on thermodynamic quantities and "positive dispersion" for Ar at $280 \mathrm{~K}$ are reported in the third section, and the last section contains conclusions of this study.

\section{Molecular dynamics simulations}

We performed MD simulations for 13 densities along the isothermal line $\mathrm{T}=280 \mathrm{~K}$ for supercritical Argon using systems of 2000 particles interacting via ab initio Woon potentials [11]. Parameters of the potentials were taken from [12] and cut-off radius was $12 \mathrm{~A}$. These potentials were the same as the used in MD simulations of supercritical Ar at $573 \mathrm{~K}[6]$. The time step in simulations was 2 fs. All the simulations were performed in microcanonical ensemble. Energy conservation was on very good level: the energy drift was less than 0.02 percent over the production runs of 480000 time steps.

Every sixth configuration was used for sampling of dynamic variables. Dynamic variables of particle density, momentum density and energy density as well as their 
time derivatives needed for GCM analysis were sampled for thirty different wave numbers directly in MD simulations. The averages of static and time correlation functions over all possible directions of different wave vectors with the same magnitude were performed.

The GCM analysis of collective dynamics in supercritical Argon was performed within a thermo-viscoelastic five-variable dynamic model:

$$
\mathbf{A}^{(5)}(k, t)=\left\{n(k, t), J^{L}(k, t), h(k, t), \dot{J}^{L}(k, t), \dot{h}(k, t)\right\}
$$

where $n(k, t), J^{L}(k, t)$ and $h(k, t)$ are the Fourier-components of hydrodynamic variables of particle density, density of longitudinal component of current and heat density, and overdots in the extended dynamic variables correspond to the first time derivatives of hydrodynamic variables. Since the static average of cross-correlations between a dynamic variable and its first time derivative is equal to zero - they are orthogonal and the extension of the hydrodynamic basis set with the time derivatives of hydrodynamic variables can be useful in describing non-hydrodynamic processes. Within the GCM approach the chosen extended set of $N$ dynamic variables is used for construction of $N \times N$ generalized hydrodynamic matrix $\mathbf{T}(k)$, eigenvalues $z_{i}(k)$ of which correspond to wave-number dependent collective modes. Analytical solution of the thermo-viscoelastic dynamic model in long-wavelength limit was obtained in [13].

The matrix elements of generalized hydrodynamic matrix $\mathbf{T}(k)$ can be expressed via wave-number dependent thermodynamic quantities, therefore for each density we estimated the following generalized thermodynamic quantities: generalized thermal expansion coefficient $\alpha_{T}(k)$, specific heat at constant volume $C_{v}(k)$, ratio of specific heats $\gamma(k)$, as well as regular static structure factor $S(k)$. We performed a check of the calculated thermodynamic quantities with the NIST database [14]. The long-wavelength limit of $C_{v}(k)$ was in perfect agreement with values $C_{v}$ estimated from temperature fluctuations in the standard way. In Figure 1 one can see that the values of $C_{v}$ calculated in MD are in good agreement with the NIST data. The values of ratio of specific heats $\gamma$ are within the acceptable $5 \%$ discrepancy with the NIST data, the calculated density dependence correctly reproduces the location of maximum. Note, that for $C_{p}$ the density dependence has its maximum at $n^{*}=0.355$, i.e. for supercritical Argon at $280 \mathrm{~K}$ the Widom line crosses the density dependences at $n^{*}=0.355$. For convenience in comparison with Lennard-Jones systems the reduced density was taken as $n^{*}=n *(3.405 \AA)^{3}$.

Adiabatic speed of sound $c_{s}$ was calculated from the long-wavelength limit of $\sqrt{\gamma(k) / S(k)}$. In Figure 2 the calculated density dependence of adiabatic speed of sound for supercritical Ar at $280 \mathrm{~K}$ is shown to be in good agreement with NIST data. The adiabatic speed of sound increases monotonically with density having the most steep increase at high densities. An analytical expression for the density dependence of adiabatic speed of sound can be found in Ref.[15]. Another important quantity that usually is used in GCM analysis of collective dynamics is the high-frequency speed of sound $c_{\infty}$, that was estimated from the following expression

$$
\lim _{k \rightarrow 0} \frac{\left\langle\dot{J}^{L}(-k) \dot{J}^{L}(k)\right\rangle}{\left\langle J^{L}(-k) J^{L}(k)\right\rangle} \propto c_{\infty}^{2} k^{2} .
$$

In Figure 2 it is shown, that in contrast to adiabatic speed of sound the $c_{\infty}$ increases almost linearly with density up to $n^{*} \sim 0.4$, while for high-density fluids it increases more steep with density. 


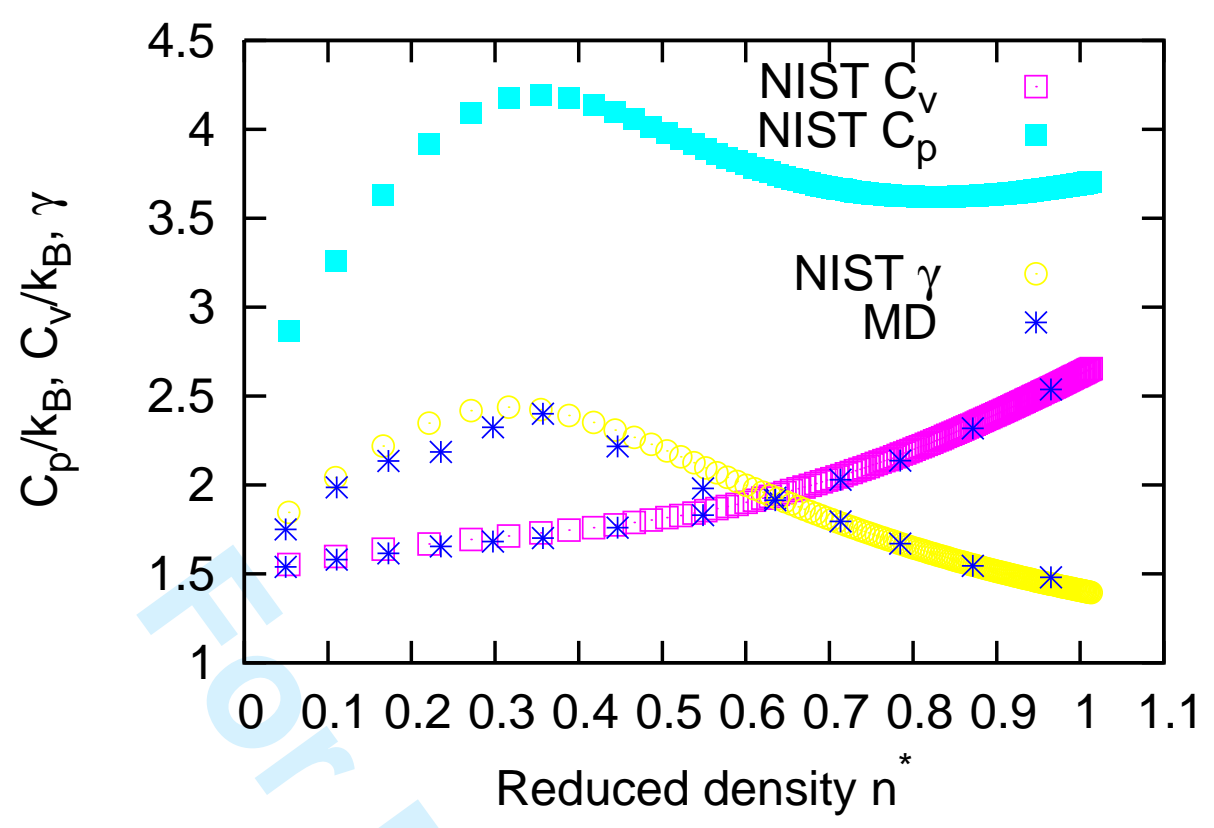

Figure 1. Specific heat at constant volume $C_{v}$ and ratio of specific heats $\gamma$ for Ar at $280 \mathrm{~K}$ calculated in this study (stars) in comparison with NIST data[14]. Dimensionless density was taken as $n^{*}=n *(3.405 \AA)^{3}$.

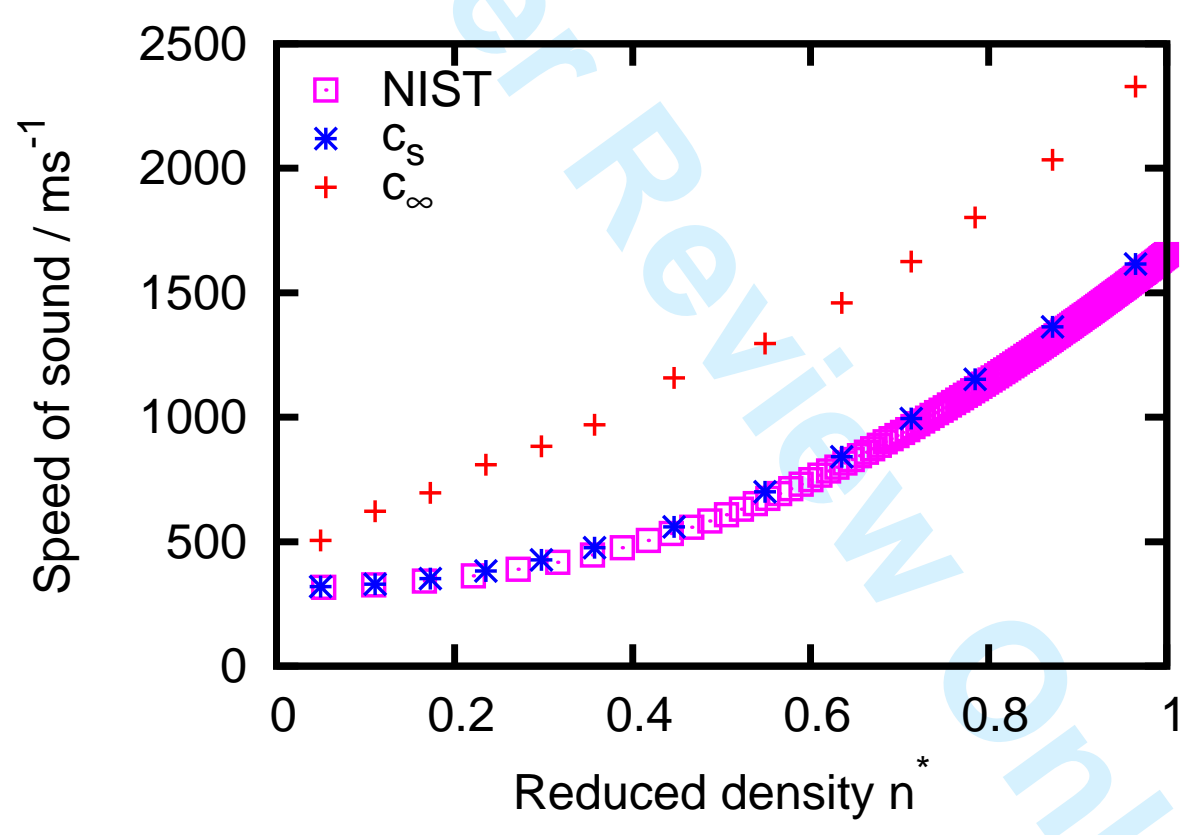

Figure 2. Calculated adiabatic (stars) and high-frequency (plus symbols) speeds of sound. By open boxes is shown the adiabatic speed of sound for Ar at $280 \mathrm{~K}$ from NIST database.

\section{Results and discussion}

We are mainly interested in dispersion of generalized sound excitations in supercritical Ar. In Figures 3 and 4 are shown the dispersion and damping of collective excitations for two densities, obtained from the imaginary and real parts of complex eigenvalues in the GCM analysis of density-density, density-energy and energy-energy hydrodynamic time correlation functions obtained in MD simula- 
tions. Matrix elements of the $5 \times 5$ generalized hydrodynamic matrix $\mathbf{T}(k)$, constructed on the basis set of dynamic variables (1), were calculated via static and time correlation functions for each wave number. No fit parameters were used. The solution of the eigenvalue problem for $\mathbf{T}(k)$ correspond to dynamic eigenmodes that can exist in some window of frequencies and given wave number $k$ in the studied fluid. In the long-wavelength region the standard three hydrodynamic modes were recovered: real eigenvalue with long-wavelength asymptote

$$
d_{1}(k)=D_{T} k^{2}
$$

where $D_{T}$ is thermal diffusivity, and a complex-conjugated pair of eigenvalues corresponding to hydrodynamic sound:

$$
z_{s}(k)=\Gamma k^{2} \pm i c_{s} k
$$

where $\Gamma=\left(D_{L}+(\gamma-1) D_{T}\right) / 2$ is the sound damping coefficient, and $D_{L}$ is kinematic viscosity. The hydrodynamic long-wavelength asymptotes for sound excitations (3) are recovered for GCM eigenvalues as it is shown in Figures 3 and 4. One can see that the region of wave numbers where the GCM sound eigenvalues reach the hydrodynamic asymptotes depends on density: for high-density fluids it is quite narrow and it increases with reduction of density. This is an evidence of different strength of processes responsible for emergence of "positive dispersion" of collective excitations.

One has to note, that in the long-wavelength region the imaginary parts of complex eigenvalues $\operatorname{Im} z_{s}(k)$ exactly correspond to the frequency of the side peak of dynamic structure factor $S(k, \omega)$. However beyond the hydrodynamic regime the side peak position is not exactly equal to $\operatorname{Im} z_{s}(k)$ because of other contributions to $S(k, \omega)$ like the asymmetric (non-Lorentzian) one, that increases linearly with $k$ in the longwavelength region and becomes quite important beyond hydrodynamic regime [? ]. Besides, non-hydrodynamic excitations like heat waves or "fast sound" in binary melts with disparate masses make non-zero contributions to the dynamic structure factors measured in scattering experiments. Experimental estimation of sound dispersion via Brillouin peak position of $S(k, \omega)$ is based on solely hydrodynamic picture of collective dynamics, while more sophisticated models are needed for analysis of experimental $S(k, \omega)$ with the purpose of estimation of true sound dispersion.

Among the five eigenmodes, obtained in the long-wavelength region two real eigenvalues tend to non-zero values in $k \rightarrow 0$ limit, that is an evidence that they belong to non-hydrodynamic processes with finite lifetime on macroscopic distances, i.e. they do not survive on macroscopic scale in comparison with hydrodynamic processes. Analytical solution for the five-variable dynamic model was obtained in long-wavelength limit in [13], and expressions for the two long-wavelength nonhydrodynamic (kinetic) modes read:

$$
d_{2}(k)=d_{2}^{0}-D_{L} k^{2}+(\gamma-1) \Delta k^{2},
$$

and

$$
d_{3}(k)=d_{3}^{0}-\gamma D_{T} k^{2}-(\gamma-1) \Delta k^{2}
$$




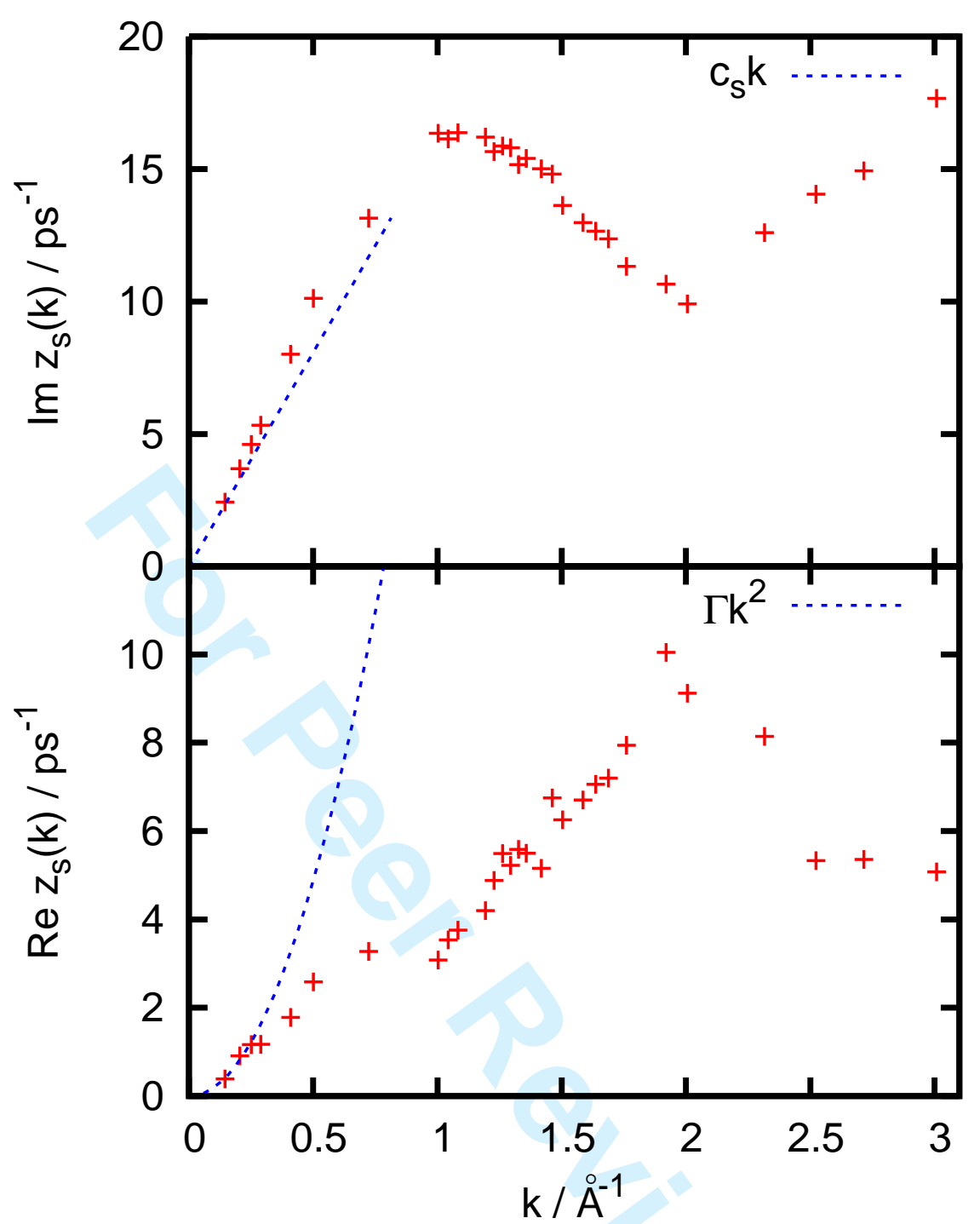

Figure 3. Dispersion and damping of generalized sound excitations for reduced density $n^{*}=0.9648$. Dashed lines show the asymptotic hydrodynamic behavior of dispersion and damping with calculated coefficients $c_{s}=16.158 \AA / p s$ and $\Gamma=18.5 \mathrm{~A}^{2} / p s$.

where the $k=0$ values are:

$$
d_{2}^{0}=\frac{c_{\infty}^{2}-c_{s}^{2}}{D_{L}}
$$

and

$$
d_{3}^{0}=\frac{c_{V}}{m \lambda}\left[G^{h}-\frac{(\gamma-1)}{\kappa_{T}}\right]
$$

Here

$$
\Delta=\frac{d_{2}^{0} d_{3}^{0}}{d_{3}^{0}-d_{2}^{0}} \frac{D_{T}}{D_{L} c_{s}^{2}}\left(D_{T}-D_{L}\right)^{2}
$$

is a coefficient that reflects coupling between the two non-hydrodynamic modes. 


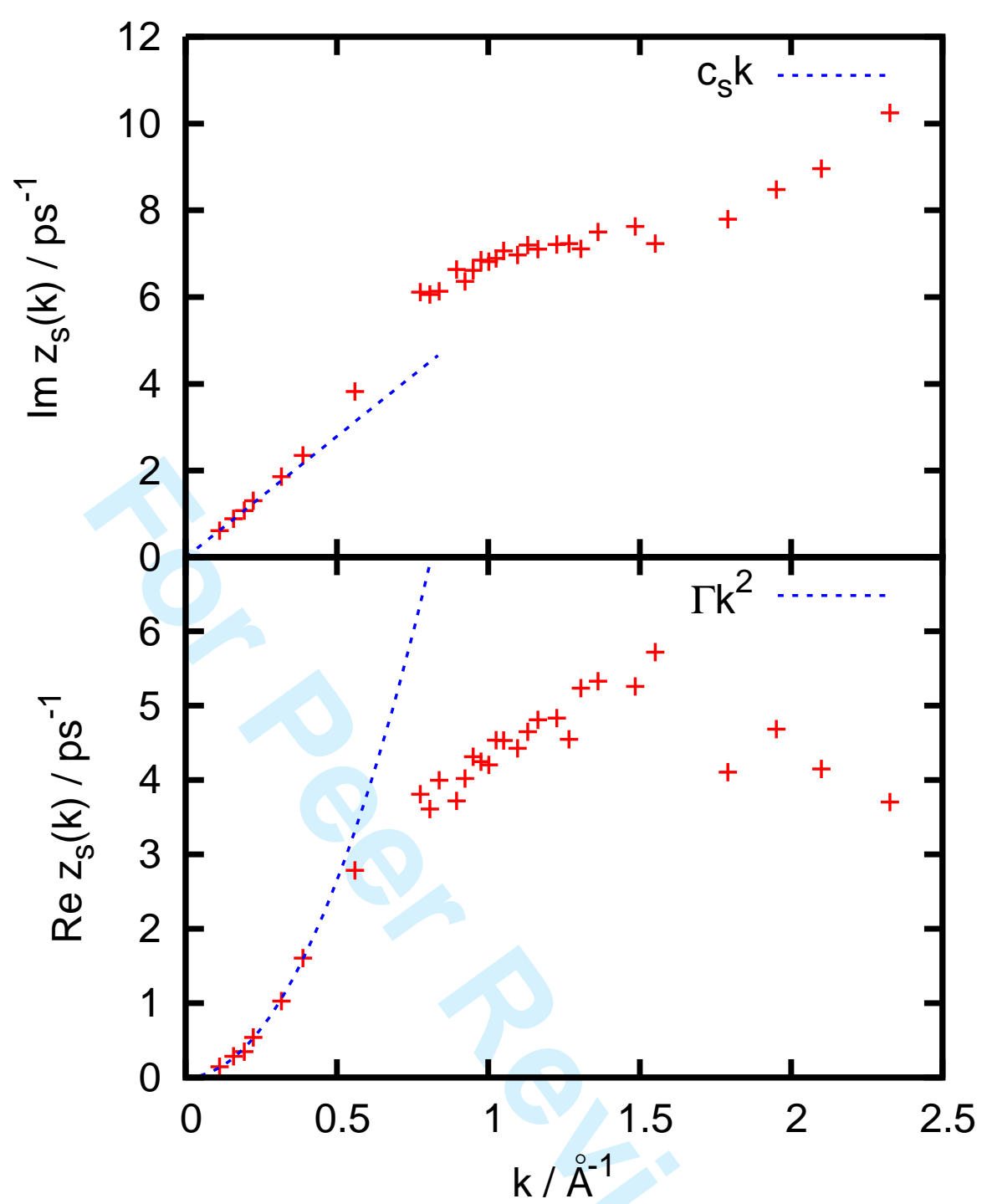

Figure 4. Dispersion and damping of generalized sound excitations for reduced density $n^{*}=0.4465$ Dashed lines show the asymptotic hydrodynamic behavior of dispersion and damping with calculated coefficients $c_{s}=5.584 \AA / p s$ and $\Gamma=10.6 \mathrm{~A}^{\circ} / p s$.

The quantities $G^{h}$ and $\kappa_{T}$ correspond to heat rigidity modulus and isothermal compressibility, respectively. Because of the factor $(\gamma-1)$ the last terms in right hand sides of (4) and (5) appear only due to coupling between the heat and density fluctuations. The quantity $\left(c_{\infty}^{2}-c_{s}^{2}\right)$ is called in the literature as the strength of structural relaxation and for wave numbers in the vicinity of the first sharp diffraction peak it is possible to show that the $d_{2}(k)$ is the inverse relaxation time of the cage of nearest neighbors[17]. Namely the non-hydrodynamic mode $d_{2}(k)$ reflects the structural relaxation in fluids. The other non-hydrodynamic mode, $d_{3}(k)$, is of thermal origin, it keeps information about heat rigidity in the fluid, and as it was shown in [18] the non-hydrodynamic processes $d_{3}(k)$ for increasing wave numbers is responsible for emergence of non-hydrodynamic heat waves that can exist on nanoscales but not on macroscopic distances and times.

The "positive dispersion" of collective excitations appears mainly due to interaction of acoustic modes with structural relaxation on the boundary of hydrodynamic regime. In Figure 5 we show how the strength of structural relaxation changes 


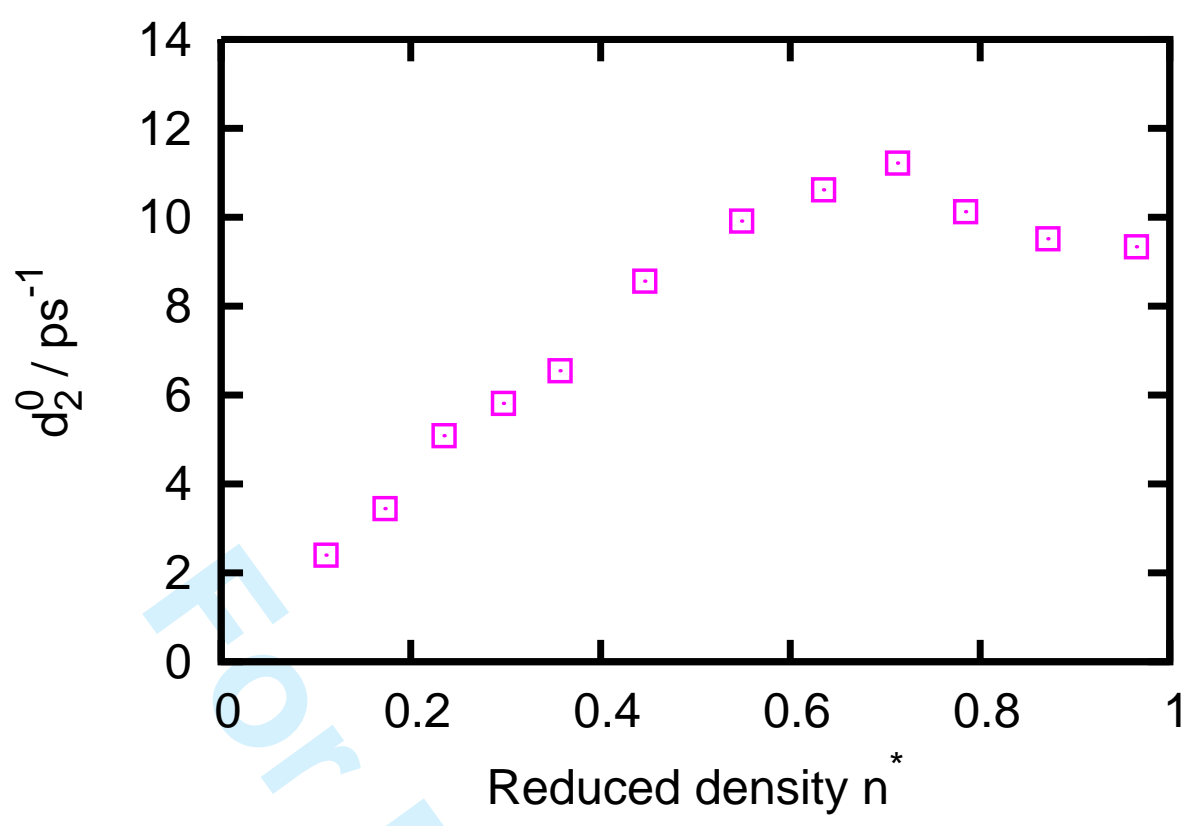

Figure 5. Dependence of the long-wavelength limit of structural relaxation mode on density for supercritical Ar at $280 \mathrm{~K}$.

with density. In the high-density region there is a maximum that appears because of more fast drop of kinematic viscosity $D_{L}$ with reduction of density than the change in $\left(c_{\infty}^{2}-c_{s}^{2}\right)$. Further decrease of density leads to monotonic decay of the $d_{2}^{0}$ that implies that even for low density fluids the non-hydrodynamic mode $d_{2}(k)$ will have quite large relaxation time and therefore will be important in collective dynamics of low-density fluids too. On the other hand the elastic properties of the system monotonically decay with decrease of density, that is seen from Figure 6, where the high-frequency shear module $G_{\infty}$ and bulk module $K_{\infty}$ are shown.

Within the GCM theory the "positive dispersion" can be obtained as a longwavelength correction to hydrodynamic dispersion law that is proportional to $k^{3}[8]$

$$
\omega_{s}(k) \approx c_{s} k+\beta k^{3}+\ldots
$$

with the prefactor $\beta$ that reads:

$$
\beta=\frac{c_{s} D_{L}^{2}}{8} \frac{5-\left(c_{\infty} / c_{s}\right)^{2}}{c_{\infty}^{2}-c_{s}^{2}}-(\gamma-1) D_{T}\left[\frac{6 D_{L}+(\gamma-5) D_{T}}{8 c_{s}}-\frac{c_{s}}{2 d_{3}^{0}}\right]
$$

For viscoelastic approximation, when the ratio of specific heats $\gamma=1$, one obtains very simple expression for the dispersion of collective excitations on the boundary of hydrodynamic regime:

$$
\omega(k) \approx c_{s} k+\frac{c_{s} D_{L}^{2}}{8} \frac{5-\left(c_{\infty} / c_{s}\right)^{2}}{c_{\infty}^{2}-c_{s}^{2}} k^{3}
$$

It follows from this expression that depending on the ratio of high-frequency speed of sound to adiabatic speed of sound one can obtain even vanishing to zero "positive dispersion" and possible "negative dispersion". From Figure 2 one can see that the largest ratio of the high-frequency speed of sound to adiabatic one $c_{\infty} / c_{s}$ larger than 2 takes place between densities $n^{*}=0.4465$ and $n^{*}=0.2351$, that implies 
almost vanishing "positive dispersion" in that region of densities. In Figure 7 one can see how the positive dispersion reduces with decrease of density. According to the NIST database for Argon [14] the Widom line crosses the isotherm $T=280 \mathrm{~K}$ at $n^{*}=0.357$ that is in agreement with Figure 7, where the "positive dispersion" almost vanishes by approaching that density. Another interesting finding is a little increase of the "positive dispersion" for very low-density fluids, that within the adopted approximation in our GCM analysis is explained by decreasing for very low density systems ratio between the high-frequency sound and adiabatic one. Note that the analysis of IXS experiments on $\mathrm{Ar}$ at $573 \mathrm{~K}$ in [6] gave evidence of a small $(\sim 4 \%)$ "positive dispersion" even in the low-density region.

\section{Conclusions}

We performed a combined study based on MD simulations of supercritical Ar along the isothermal line of $280 \mathrm{~K}$ and theoretical GCM analysis of collective excitations. We made check that our calculated density dependences of adiabatic speed of sound, ratio of specific heats and specific heat at constant volume were in good agreement with the NIST data. Based on these quantities we studied the "positive dispersion" of collective excitations in supercritical Ar and how it depends on density.

We made use of the five-variable dynamic model for GCM analysis of time correlation functions obtained in MD simulations. We found, that the "positive dispersion" reduces with the decrease of density. Theoretical GCM expression for the correction to hydrodynamic dispersion law yields "positive dispersion" in good agreement with MD data in a wide region of densities. We found, that the theoretical expression for "positive dispersion" is in agreement with a suggestion that the "positive dispersion" can vanish in the vicinity of the Widom line [6]. 
Figure 7. Density dependence of the factor $\beta$ (6) for "positive dispersion" of collective excitations in supercritical Ar at $280 \mathrm{~K}$ as calculated within the viscoelastic approximation.

\section{Acknowledgements}

TB was partially supported by the project PHA-0107U002081.

\section{References}

[1]W. Montfrooij and I. De Schepper, Excitations in Simple Liquids, Liquid Metals and Superfluids, (University Press, Oxford, 2010)

[2] T. Scopigno, G. Ruocco and F. Sette, Rev. Mod. Phys. 77 (3), 881 (2005).

[3] M.H. Ernst, J.R. Dorfman, J.Stat.Phys., 12, 311 (1975).

[4]J.Bosse, W.Götze and M.Lücke, Phys. Rev. A, 18, 1176 (1978)

[5]I.M.deSchepper, P.Verkerk, A.A. van Well, L.A. deGraaf, Phys.Lett. A, 104, 29 (1984).

[6] G. Simeoni, T. Bryk, F.A. Gorelli, M. Krisch, G. Ruocco, M. Santoro and T. Scopigno, Nature Phys. 6 (7), $503(2010)$.

[7]P.F. McMillan and H.E. Stanley, Nature Phys. 6 (7), 479 (2010).

[8]T. Bryk, I. Mryglod, T. Scopigno, G. Ruocco, F. Gorelli and M. Santoro, J.Chem.Phys. 133, 024502 $(2010)$.

[9] I.M.deSchepper, E.G.D.Cohen, C.Bruin, J.C. van Rijs, W.Montfrooij, and L.A. de Graaf, Phys. Rev. A 38, 271 (1988).

[10]I.M.Mryglod, I.P.Omelyan, and M.V.Tokarchuk, Mol. Phys. 84, 235 (1995)

[11]D.E.Woon, Chem.Phys.Lett. 204, 29 (1993).

[12] J.-M.Bomont, J.-L.Bretonnet, T.Pfleiderer and H.Bertagnolli, J. Chem. Phys. 113, 6815 (2000).

[13]T. Bryk and I. Mryglod, Condens. Matter Phys. 7, (3) 471 (2004).

[14]E.W. Lemmon, M.O. McLinden and D.G. Friend, "Thermophysical Properties of Fluid Systems" in NIST Chemistry WebBook, NIST Standard Reference Database Number 69, edited by P.J. Linstrom and W.G. Mallard, (National Institute of Standards and Technology, Gaithersburg MD, 20899) http://webbook.nist.gov

[15] M.Schoen and F.Porcheron, Phys. Rev. E 67, 051202 (2003).

[18] T. Bryk and I. Mryglod, Phys. Rev. E 64, (3) 032202 (2001).

[17]T. Bryk and I. Mryglod, Condens. Matter Phys. 11, (1) 139 (2008).

[18]T. Bryk and I. Mryglod, Phys. Rev. E 63, (5) 051202 (2001). 